International Journal of Pure and Applied Mathematics

Volume 114 No. $3 \quad 2017,647-658$

ISSN: 1311-8080 (printed version); ISSN: 1314-3395 (on-line version)

url: http://www.ijpam.eu

doi: 10.12732/ijpam.v114i3.19

\title{
FIXED POINT FOR CYCLIC MULTI-VALUED MAPPING IN COMPLETE DISLOCATED QUASI-b-METRIC SPACES
}

\author{
Ampon Buayai ${ }^{1}$, Araya Wiwatwanich ${ }^{2}$, Annop Kaewkhao ${ }^{3} \S$ \\ $1,2,3$ Department of Mathematics \\ Faculty of Science \\ Burapha University \\ Chonburi Province 20131, THAILAND \\ Centre of Excellence in Mathematics \\ PERDO, CHE, THAILAND
}

\begin{abstract}
This paper aims to extend the concept of cyclic single-valued mapping to the case of multi-valued mapping in dislocated quasi-b-metric spaces. The existence of fixed point in dislocated quasi-b-metric spaces.
\end{abstract}

AMS Subject Classification: 47H10, 54H25

Key Words: fixed point, multi-valued mapping, cyclic multi-valued mapping, dislocated quasi-b-metric spaces

\section{Introduction and Preliminaries}

Currently, fixed point theorem (we say that $x \in X$ is a fixed point of self-map $T$ if $T x=x$ ) has been widely studied. There are many results have been published. A fundamental result of fixed point theory is Banach contraction principle, which introduced in 1922 by Banach [1] as the following theorem:

Received: $\quad$ March 4, 2017

Revised: $\quad$ May 9, 2017

Published: $\quad$ May 23, 2017

(c) 2017 Academic Publications, Ltd. url: www.acadpubl.eu

${ }^{\S}$ Correspondence author 
Theorem 1. Let $(X, d)$ be a complete metric space and let $T: X \rightarrow X$ be a Banach contraction mapping i.e., there exists a constant $a \in[0,1)$ such that

$$
d(T x, T y) \leq a d(x, y)
$$

for all $x, y \in X$. Then $T$ has a unique fixed point.

In 1969, Kannan [2] introduced the concept of Kannan mapping as follows:

Theorem 2. Let $(X, d)$ be a complete metric space and let $T$ be a Kannan mapping i.e., there exists a constant $b \in\left[0, \frac{1}{2}\right)$ such that

$$
d(T x, T y) \leq b[d(x, T x)+d(y, T y)]
$$

for all $x, y \in X$. Then $T$ has a unique fixed point.

Chatterjea [7] introduced the concept of Chatterjea mapping in 1972, as follows:

Theorem 3. Let $(X, d)$ be a complete metric space and let $T$ be a Chatterjea mapping i.e., there exists $c \in\left[0, \frac{1}{2}\right)$ such that

$$
d(T x, T y) \leq c[d(x, T y)+d(y, T x)]
$$

for all $x, y \in X$. Then $T$ has a unique fixed point.

The concept of Zamfirescu mapping was introduced in 1972 by Zamfirescu [4] as the following theorem:

Theorem 4. Let $(X, d)$ be a complete metric space and let $T$ be a $Z a m$ firescu mapping i.e., there exist real numbers $a \in[0,1), b \in\left[0, \frac{1}{2}\right)$ and $c \in\left[0, \frac{1}{2}\right)$ such that at least one of the following conditions is true:

$$
\begin{aligned}
& \left(z_{1}\right) \quad d(T x, T y) \leq a d(x, y), \\
& \left(z_{2}\right) \quad d(T x, T y) \leq b[d(x, T x)+d(y, T y)], \\
& \left(z_{3}\right) \quad d(T x, T y) \leq c[d(x, T y)+d(y, T x)] .
\end{aligned}
$$

for all $x, y \in X$. Then $T$ has a unique fixed point.

Next, we recall the concept of cyclic map as the following definition:

Definition 5. Let $A$ and $B$ be nonempty subsets of metric space $(X, d)$ and $T: A \cup B \rightarrow A \cup B$. Then $T$ is called a cyclic mapping if and only if $T(A) \subseteq B$ and $T(B) \subseteq A$.

In 2003, Kirk et al. [5] extended Banach contraction to a case of cyclic mapping as follows: 
Theorem 6. Let $A$ and $B$ be nonempty closed subsets of metric space $(X, d)$ and let a cyclic mapping $T$ be a cyclic-Banach contraction i.e., there exists $a \in[0,1)$ such that

$$
d(T x, T y) \leq a d(x, y)
$$

for all $x \in A$ and $y \in B$. Then $T$ has a unique fixed point in $A \cap B$.

In 2010, Karapinar and Erhan [6] extended the concept of Kannan mapping to cyclic mapping as the following theorem:

Theorem 7. Let $A$ and $B$ be nonempty closed subsets of metric space $(X, d)$ and let a cyclic mapping $T$ be a cyclic-Kannan contraction i.e., there exists $b \in\left[0, \frac{1}{2}\right)$ such that

$$
d(T x, T y) \leq b[d(x, T x)+d(y, T y)]
$$

for all $x \in A$ and $y \in B$. Then $T$ has a unique fixed point in $A \cap B$.

The concept of metric space has been extended, improved and generalized in many different ways (see, [7],[8],[9] ). Let $X$ be a nonempty set, a function $d: X \times X \rightarrow[0, \infty)$ is called distance on $X$. The pair $(X, d)$ is called a distance space. We need the following conditions:

$\left(d_{1}\right) \quad d(x, y)=d(y, x)=0$ implies $x=y$,

$\left(d_{2}\right) \quad d(x, y)=d(y, x)=0$ iff $x=y$,

$\left(d_{3}\right) \quad d(x, y)=d(y, x)$

$\left(d_{4}\right) \quad d(x, y) \leq s[d(x, z)+d(z, y)]$ with constant $s \geq 1$

for all $x, y, z \in X$. If $(X, d, s)$ satisfies conditions $\left(d_{2}\right),\left(d_{3}\right)$ and $\left(d_{4}\right)$, then it is called a $b$-metric space. If $(X, d, s)$ satisfies conditions $\left(d_{2}\right)$ and $\left(d_{4}\right)$, then it is called a quasi-b-metric space. If it satisfies conditions $\left(d_{1}\right),\left(d_{3}\right)$ and $\left(d_{4}\right)$, then it is called a dislocated b-metric space. If $(X, d, s)$ satisfies conditions $\left(d_{1}\right)$ and $\left(d_{4}\right)$, then it is called a dislocated quasi-b-metric space ( dqb-metric space for short), which was introduced by Klin-eam and Suanoom [10] in 2015. They also gave some examples of dqb-metric spaces:

Example 8. [10] Let $X=\mathbb{R}$ and let

$$
d(x, y)=|x-y|^{2}+\frac{|x|}{m}+\frac{|y|}{n},
$$


where $m, n \in \mathbb{N} \backslash\{1\}$ with $m \neq n$. Then $(X, d, s)$ is a dqb-metric space with the coefficient $s=2$, but $(X, d, s)$ is neither a quasi-b-metric space nor dislocated b-metric space.

Example 9. [10] Let $X=\mathbb{R}$ and let

$$
d(x, y)=|x-y|^{2}+3|x|^{2}+2|y|^{2},
$$

then $(X, d, s)$ is a dqb-metric space with the coefficient $s=2$, but $(X, d, s)$ is neither a quasi-b-metric space nor dislocated b-metric space.

Moreover, they introduced the notion of dqb-cyclic-Banach and dqb-cyclicKannan mapping and derived the fixed point theorems for such space as follows:

Theorem 10. [10] Let $A$ and $B$ be nonempty closed subsets of a complete dqb-metric space $(X, d, s)$. If $T$ is a cyclic-Banach contraction, then $T$ has a unique fixed point in $A \cap B$.

Theorem 11. [10] Let $A$ and $B$ be nonempty closed subsets of a complete dqb-metric space $(X, d, s)$. If $T$ is a cyclic-Kannan mapping,then $T$ has a unique fixed point in $A \cap B$.

In 1967, Nadler initiated the concept of multi-valued mapping. Let $(X, d, s)$ be a dqb-metric space and $A, B \in \mathcal{C B}(X)$ where $\mathcal{C B}(X)$ denotes the family of non-empty closed and bounded subsets of $X$, define the functional $H: \mathcal{C B}(X) \times$ $\mathcal{C B}(X) \rightarrow \mathbb{R}^{+}$by

$$
H(A, B)=\max \{h(A, B), h(B, A)\} .
$$

where

$$
h(A, B)=\sup \{d(a, B): a \in A\},
$$

with

$$
d(a, B)=\inf \{d(a, b): b \in B\} .
$$

In the following lemma, we list some basic properties of $H$ :

Lemma 12. Let $(X, d, s)$ be a dqb-metric space. For any $A, B \in \mathcal{C B}(X)$ and any $x, y \in X$, the following statements are true:

1. $d(x, B) \leq d(x, b) \quad$ for any $b \in B$,

2. $h(A, B) \leq H(A, B)$,

3. $d(a, B) \leq H(A, B) \quad$ for any $a \in A$,

4. $H(A, A)=0$, 
5. $H(A, B)=H(B, A)$,

6. $d(x, A) \leq s[d(x, y)+d(y, A)]$.

Later, in 1969, he studied the existence of a fixed point of a contraction multivalued mapping. An element $x \in X$ such that $x \in T x$ is called a fixed point of a multi-valued mapping $T: X \longrightarrow \mathcal{C B}(X)$. We denote by $F_{T}$ the set of all fixed point of $T$, i.e., $F_{T}=\{x \in X \mid x \in T x\}$. The following result was proved by Nadler [11]:

Theorem 13. [11] Let $(X, d)$ be metric space and $T: X \longrightarrow \mathcal{C B}(X)$ is said to be a multi-value contraction mapping, i.e., there exists a real number $a \in[0,1)$ such that

$$
H(T x, T y) \leq \operatorname{ad}(x, y)
$$

for all $x, y \in X$. Then $T$ has a fixed point.

The concept of Zamfirescu multi-valued mapping was introduced by Kaewkhao and Neammanee in 2010 [12] as follows:

Theorem 14. [12] Let $(X, d)$ be a complete metric space and $T: X \longrightarrow$ $\mathcal{C B}(X)$ be a multi-valued Zamfirescu mapping, i.e., there exist real numbers $a, b$ and $c$ satisfying $a \in[0,1), b \in\left[0, \frac{1}{2}\right)$ and $c \in\left[0, \frac{1}{2}\right)$ such that, for all $x, y \in X$, at least one of the following conditions is satisfied:

1. $H(T x, T y) \leq a d(x, y)$,

2. $H(T x, T y) \leq b[d(x, T x)+d(y, T y)]$,

3. $H(T x, T y) \leq c[d(x, T y)+d(y, T x)]$.

Then $T$ has fixed point and it is complete.

In 2011, Kaewkhao and Neammanee [13] extended a concept of cyclic singlevalue to the multi-valued and also studied the existence of a fixed point which is stated in the following theorem:

Theorem 15. [13] Let $A$ and $B$ be non-empty closed subsets of a complete metric space $(X, d)$ and let $T$ be a cyclic multi-valued mapping with closed and bounded value i.e., there exists a constant $a \in[0,1)$ such that

$$
H(T x, T y) \leq a d(x, y)
$$

for all $x \in A, y \in B$. Then $T$ has at least one fixed point in $A \cap B$. 
Recently, Jinakul et al. [14] have studied the conditions for existence of a common fixed point of any two multi-valued mappings on a complete b-metric space. The result was presented as follows:

Theorem 16. Let $(X, d, s)$ be a complete b-metric space and let $S, T ; X \rightarrow$ $\mathcal{C B}(X)$ be multi-valued mappings satisfying

$$
H(T x, S y) \leq a d(x, T y)+b(d(x, S y)+d(T y, T x))
$$

, where $a+2 b s<1, a, b \geq 0, b<\frac{1}{s^{2}}$ for all $x, y \in X$. Then $F(T)=F(S) \neq \phi$ and $T x=S x=F(T)$ for all $x \in F(T)$.

In this paper, we extend the concept of cyclic single-valued mapping to the case of multi-valued mapping and derive the existence of fixed point in dqbmetric.

Now, we recall definitions about convergence of sequences, Cauchy sequence, closeness, boundedness and completeness in a dqb-metric space as follows:

Definition 17. Let $(X, d, s)$ be a dqb-metric space.

(1) A sequence $\left\{x_{n}\right\} d q b$-converges to $x \in X$ if and only if for all $\epsilon>0$ there exist $n_{\epsilon} \in \mathbb{N}$ such that for all $n \geq n_{\epsilon}, d\left(x, x_{n}\right)<\epsilon$. In this case, we write $\lim _{n \rightarrow \infty} d\left(x, x_{n}\right)=0$.

(2) A sequence $\left\{x_{n}\right\}$ is called a dqb-Cauchy sequence if and only if for all $\epsilon>0$ there exist $n_{\epsilon} \in \mathbb{N}$ such that for all $m>n \geq n_{\epsilon}, d\left(x_{n}, x_{m}\right)<\epsilon$. In this case, we write $\lim _{n \rightarrow \infty} d\left(x_{n}, x_{m}\right)=0$.

(3) A dqb-metric space $(X, d, s)$ is complete if every Cauchy sequence in $X$ is convergent in $X$.

(4) A subset $Y$ of $X$ is closed if and only if for each sequence $\left\{x_{n}\right\}$ in $Y$ which converges to an element $x$, we have $x \in Y$.

(5) A subset $Y$ of $X$ is bounded if there exists $M \in(0, \infty)$ such that $d(x, y) \leq$ $M$ for all $x, y \in Y$.

The following results are useful for some of the proof in the paper:

Lemma 18. Let $(X, d, s)$ be a dqb-metric space and let $A$ be a nonempty closed subset of $X$ and $x \in X$. If $d(x, A)=0$, then $x \in A$.

Proof. Suppose $d(x, A)=0$, we have $\inf \{d(x, a): a \in A\}=0$. For $n \in \mathbb{N}$, we have $\frac{1}{n}>0$, then there exists a sequence $\left\{a_{n}\right\}$ in $A$ such that $d\left(x, a_{n}\right)<\frac{1}{n}$. 
Next, we will show that a sequence $\left\{a_{n}\right\}$ dqb-converges to $x$. Let $\epsilon>0$, by the Archimedean property there exists $N \in \mathbb{N}$ such that $\frac{1}{N}<\epsilon$. Then for each $n \in \mathbb{N}$ such that $n \geq N$, we arrive at $\frac{1}{n} \leq \frac{1}{N}$. Thus $d\left(x, a_{n}\right)<\frac{1}{n} \leq \frac{1}{N}<\epsilon$. Hence a sequence $\left\{a_{n}\right\}$ dqb-converges to $x$. Since $A$ is closed, then we have $x \in A$.

Lemma 19. Let $(X, d, s)$ be a dqb-metric space and $A, B \in \mathcal{C B}(X)$. If $H(A, B)=0$, then $A=B$.

Proof. Suppose that $H(A, B)=0$, we have $\max \{h(A, B), h(B, A)\}=0$, which implies that $h(A, B)=0$ and $h(B, A)=0$. Since $h(A, B)=0$, then $d(a, B)=0$, for all $a \in A$. By Lemma 18, we have $a \in B$, i.e., $A \subset B$. Similarly, we also have $A \subset B$. Thus $A=B$.

Lemma 20. Let $(X, d, s)$ be a dqb-metric space and $A, B \in \mathcal{C B}(X)$. Then for each $q>1$ and for all $x \in A$ there exists $y \in B$ such that $d(x, y) \leq q H(A, B)$.

Proof. Let $q>1$ and $x \in A$. By the definition of infimum, we have there exists $y \in B$ such that

$$
d(x, y) \leq q d(x, B) \leq q h(A, B) \leq q H(A, B) .
$$

\section{Main Results}

In this section, we prove the existence of fixed point for cyclic multi-valued Banach contraction mappings and cyclic multi-valued Kannan mappings in dqbmetric spaces.

Theorem 21. Let $A$ and $B$ be non-empty closed subsets of a complete dqb-metric space $(X, d, s)$ and $T: A \cup B \rightarrow \mathcal{C B}(X)$ be a cyclic multi-valued mapping. If there exists a constant $a \in(0,1)$ with $s a<1$ such that

$$
H(T x, T y) \leq a d(x, y),
$$

for all $x \in A$ and $y \in B$. Then $T$ has at least one fixed point in $A \cap B$.

Proof. Let $1<q<\frac{1}{a}$ and let $x_{0} \in A$ be fixed. Choose $x_{1} \in T x_{0} \subseteq B$. By Lemma 20, there exists $x_{2} \in T x_{1} \subseteq A$ such that

$$
\begin{aligned}
d\left(x_{1}, x_{2}\right) & \leq q H\left(T x_{0}, T x_{1}\right) \\
& \leq q a d\left(x_{0}, x_{1}\right)
\end{aligned}
$$


Again by Lemma 20, there exists $x_{3} \in T x_{2} \subseteq B$ such that

$$
\begin{aligned}
d\left(x_{2}, x_{3}\right) & \leq q H\left(T x_{1}, T x_{2}\right) \\
& \leq q a d\left(x_{1}, x_{2}\right) \\
& \leq(q a)^{2} d\left(x_{0}, x_{1}\right)
\end{aligned}
$$

Continuing this process, for $n \in \mathbb{N}$ we have $x_{n+1} \in T x_{n}$ such that

$$
\begin{aligned}
d\left(x_{n}, x_{n+1}\right) & \leq(q a)^{n} d\left(x_{0}, x_{1}\right) \\
& =\alpha^{n} d\left(x_{0}, x_{1}\right), \text { where } \alpha=q a<1 .
\end{aligned}
$$

Next, we will show that the sequence $\left\{x_{n}\right\}$ is dqb-Cauchy sequence. Let $m, n \in$ $\mathbb{N}$ with $m=n+k$ for some $k \in \mathbb{N}$, by using the triangular inequality, we have

$$
\begin{aligned}
d\left(x_{n}, x_{m}\right) & =d\left(x_{n}, x_{n+k}\right) \\
& \leq s d\left(x_{n}, x_{n+1}\right)+s^{2} d\left(x_{n+1}, x_{n+2}\right)+\cdots+s^{k} d\left(x_{n+k-1}, x_{n+k}\right) \\
& \leq s \alpha^{n} d\left(x_{0}, x_{1}\right)+s^{2} \alpha^{n+1} d\left(x_{0}, x_{1}\right)+\cdots+s^{k} \alpha^{n+k-1} d\left(x_{0}, x_{1}\right) \\
& =\left[1+(s \alpha)+(s \alpha)^{2}+\cdots+(s \alpha)^{k-1}\right] s \alpha^{n} d\left(x_{0}, x_{1}\right) \\
& =\left[\frac{1-(s \alpha)^{k}}{1-s \alpha}\right] s \alpha^{n} d\left(x_{0}, x_{1}\right) .
\end{aligned}
$$

Since $\alpha<1$, then, we get $\lim _{n \rightarrow \infty} d\left(x_{n}, x_{m}\right)=0$. Hence $\left\{x_{n}\right\}$ is dqb-Cauchy sequence. Since $(X, d, s)$ is complete, then sequence $\left\{x_{n}\right\}$ dqb-converges to some $x \in X$. We note that $\left\{x_{2 n}\right\}$ is a sequence in $A$ and $\left\{x_{2 n-1}\right\}$ is a sequence in $B$ in a way that both sequences tend to the same limit $x$. Since $A$ and $B$ are closed, so $x \in A \cap B$. Next, we show that $x \in T x$. Consider

$$
\begin{aligned}
d(x, T x) & \leq s\left[d\left(x, x_{n+1}\right)+d\left(x_{n+1}, T x\right)\right] \\
& =s d\left(x, x_{n+1}\right)+s d\left(x_{n+1}, T x\right) \\
& \leq s d\left(x, x_{n+1}\right)+s H\left(T x_{n}, T x\right) \\
& =s d\left(x, x_{n+1}\right)+s H\left(T x, T x_{n}\right) \\
& \leq s d\left(x, x_{n+1}\right)+\operatorname{sad}\left(x, x_{n}\right) .
\end{aligned}
$$

Since sequence $\left\{x_{n}\right\}$ dqb-converges to $x$, then, we get $\lim _{n \rightarrow \infty} d(x, T x)=0$. Hence $d(x, T x)=0$. Since $T x$ is closed and by Lemma 18, we have $x \in T x$. Therefore $T$ has at least one fixed point in $A \cap B$ as required.

Finally, we introducing the concept of cyclic multi-valued Kannan mapping in dqb-metric space: 
Theorem 22. Let $A$ and $B$ be non-empty closed subsets of a complete dqb-metric space $(X, d, s)$ and $T: A \cup B \rightarrow \mathcal{C B}(X)$ be a cyclic multi-valued mapping. If there exists a constant $b \in\left(0, \frac{1}{2}\right)$ with $s b<\frac{1}{2}$ such that

$$
H(T x, T y) \leq b[d(x, T x)+d(y, T y)]
$$

for all $x \in A$ and $y \in B$. Then $T$ has at least one fixed point in $A \cap B$.

Proof. Let $1<q<\frac{1}{2 b}$ and let $x_{0} \in A$ be fixed. Choose $x_{1} \in T x_{0} \subseteq B$. By Lemma 20, there exists $x_{2} \in T x_{1} \subseteq A$ such that

$$
\begin{aligned}
d\left(x_{1}, x_{2}\right) & \leq q H\left(T x_{0}, T x_{1}\right) \\
& \leq q b\left[d\left(x_{0}, T x_{0}\right)+d\left(x_{1}, T x_{1}\right)\right] \\
& \leq q b d\left(x_{0}, x_{1}\right)+q b d\left(x_{1}, x_{2}\right),
\end{aligned}
$$

so

$$
d\left(x_{1}, x_{2}\right) \leq \frac{q b}{1-q b} d\left(x_{0}, x_{1}\right)
$$

Again by Lemma 20, there exists $x_{3} \in T x_{2} \subseteq B$ such that

$$
\begin{aligned}
d\left(x_{2}, x_{3}\right) & \leq q H\left(T x_{1}, T x_{2}\right) \\
& \leq q b\left[d\left(x_{1}, T x_{1}\right)+d\left(x_{2}, T x_{2}\right)\right] \\
& \leq q b d\left(x_{1}, x_{2}\right)+q b d\left(x_{2}, x_{3}\right),
\end{aligned}
$$

so

$$
\begin{aligned}
d\left(x_{2}, x_{3}\right) & \leq \frac{q b}{1-q b} d\left(x_{1}, x_{2}\right) \\
& \leq\left(\frac{q b}{1-q b}\right)^{2} d\left(x_{0}, x_{1}\right) .
\end{aligned}
$$

Continuing this process, for $n \in \mathbb{N}$ we have $x_{n+1} \in T x_{n}$ such that

$$
\begin{aligned}
d\left(x_{n}, x_{n+1}\right) & \leq\left(\frac{q b}{1-q b}\right)^{n} d\left(x_{0}, x_{1}\right) \\
& =\beta^{n} d\left(x_{0}, x_{1}\right) \text { where } \beta=\frac{q b}{1-q b}<1 .
\end{aligned}
$$


Next, we will show that the sequence $\left\{x_{n}\right\}$ is dqb-Cauchy sequence. Let $m, n \in$ $\mathbb{N}$ with $m=n+k$, by using the triangular inequality, we have

$$
\begin{aligned}
d\left(x_{n}, x_{m}\right) & =d\left(x_{n}, x_{n+k}\right) \\
& \leq s d\left(x_{n}, x_{n+1}\right)+s^{2} d\left(x_{n+1}, x_{n+2}\right)+\cdots+s^{k} d\left(x_{n+k-1}, x_{n+k}\right) \\
& \leq s \beta^{n} d\left(x_{0}, x_{1}\right)+s^{2} \beta^{n+1} d\left(x_{0}, x_{1}\right)+\cdots+s^{k} \beta^{n+k-1} d\left(x_{0}, x_{1}\right) \\
& =\left[1+(s \beta)+(s \beta)^{2}+\cdots+(s \beta)^{k-1}\right] s \beta^{n} d\left(x_{0}, x_{1}\right) \\
& =\left[\frac{1-(s \beta)^{k}}{1-s \beta}\right] s \beta^{n} d\left(x_{0}, x_{1}\right) .
\end{aligned}
$$

Since $\beta<1$, then, we get $\lim _{n \rightarrow \infty} d\left(x_{n}, x_{m}\right)=0$. Hence $\left\{x_{n}\right\}$ is dqb-Cauchy sequence. Since $(X, d, s)$ is complete, then sequence $\left\{x_{n}\right\}$ dqb-converges to some $x \in X$. We note that $\left\{x_{2 n}\right\}$ is a sequence in $A$ and $\left\{x_{2 n-1}\right\}$ is a sequence in $B$ in a way that both sequences tend to the same limit $x$. Since $A$ and $B$ are closed, we have $x \in A \cap B$. Next, we show that $x \in T x$. Consider

$$
\begin{aligned}
d(x, T x) & \leq s d\left(x, x_{n+1}\right)+s d\left(x_{n+1}, T x\right) \\
& \leq s d\left(x, x_{n+1}\right)+s H\left(T x_{n}, T x\right) \\
& \leq s d\left(x, x_{n+1}\right)+s b\left[d\left(x_{n}, T x_{n}\right)+d(x, T x)\right] \\
& \leq s d\left(x, x_{n+1}\right)+\operatorname{sbd}\left(x_{n}, x_{n+1}\right)+\operatorname{sbd}(x, T x) \\
& \leq s d\left(x, x_{n+1}\right)+\operatorname{sb} \beta^{n} d\left(x_{0}, x_{1}\right)+\operatorname{sbd}(x, T x) .
\end{aligned}
$$

So

$$
d(x, T x) \leq \frac{s}{1-s b} d\left(x, x_{n+1}\right)+\frac{s b}{1-s b} \beta^{n} d\left(x_{0}, x_{1}\right)
$$

Since sequence $\left\{x_{n}\right\}$ dqb-converges to $x$ and $\beta<1$, then, we get $\lim _{n \rightarrow \infty} d(x, T x)=$ 0 . Hence $d(x, T x)=0$. Since $T x$ is closed and by Lemma 18 , we have $x \in T x$. Therefore $T$ has at least one fixed point in $A \cap B$ as required.

The following corollary can be considered as a particular case of Theorem 21 if we take $s=1$ :

Corollary 23. Let $A$ and $B$ be non-empty closed subsets of a complete dqb-metric space $(X, d, 1)$ and let $T$ be a cyclic multi-valued mapping with closed and bounded value i.e., there exists a constant $a \in[0,1)$ such that

$$
H(T x, T y) \leq a d(x, y),
$$

for all $x \in A, y \in B$. Then $T$ has at least one fixed point in $A \cap B$. 
Notice that Corollary 23 is in fact the previous result on complete metric space $(X, d)$ proved by Kaewkhao and Neammanee [13].

\section{Acknowledgements}

The authors would like to thank the Faculty of Science Burapha University for providing fond to support the research. We are also grateful to the reviewers for comments that greatly improved the manuscript.

\section{References}

[1] S. Banach, Sur les opérations dans les ensembles abstraits et leur application aux équations intégrales, Fundam. Math., 3 (1922), 133-181.

[2] R. Kannan, Some results on fixed points-II. The Amer. Math. Monthly., 76(4) (1969), 405-408, doi: $10.2307 / 2316437$.

[3] S. K. Chatterjea, Fixed-point theorems. Comptes Rendus de l'Académie Bulgare des Sciences. 25 (1972), 727-730.

[4] T. Zamfirescu, Fixed point theorems in metric spaces. Archiv der Mathematik (Basel), 23 (1972), 292-293, doi: 10.1007/BF01304884.

[5] W. A. Krik, P. S. Srinivasan, P. Veeramani, Fixed points for mapping satisfying cyclic contractive conditions. Fixed point Theory., 4(1) (2013), 79-89.

[6] E. Karapinar, I. M. Erhan, Best proximity on different type contractions. Applied Mathematics \& Information Sciences., 5 (2010), 558-569.

[7] S. Czerwik, Nonlinear set-valued contraction mappings in b-metric spaces, Atti Semin. Mat. Fis. Univ. Modena., 46(3) (1998), 263-276.

[8] M. H. Shah, N. Hussain, Nonlinear contractions in partially ordered quasi b-metric spaces. Communications of the Korean Mathematical Society. 27(1) (2012), 117-128, doi: 10.4134/CKMS.2012.27.1.117.

[9] M. A. Alghamdi, N. Hussain, P. Salimi, Fixed point and coupled fixed point theorems on b-metric-like spaces. Journal of Inequalities and Applications., (2013), 402, doi: 10.1186/1029-242X-2013-402.

[10] C. Klin-Eam, C. Suanoom, Dislocated quasi-b-metric spaces and fixed point theorems for cyclic contractions, Fixed Point Theory and Applications (2015), doi: 10.1186/s13663015-0325-2.

[11] S.B. Nadler, Multi-valued contraction mappings. Pacific Journal of Mathematics., 30(2) (1969), 475-488.

[12] A. Kaewkhao, K. Neammanee, Fixed point Theorems of multi-valued Zamfirescu mapping. Journal of Mathematics Research., 2(2) (2010), 150-156, doi: 10.5539/jmr.v2n2p150. 
[13] A. Kaewkhao, K. Neammanee, Fixed points and best proximity points for multi-valued mapping satisfying cyclical condition. International Journal of Mathematical Sciences 86 Applications., 1(1) (2011).

[14] C. Jinakul, A. Wiwatwanich, A. Kaewkhao, Common fixed point theorem for multivalued mappings on b-metric spaces. International Journal of Pure and Applied Mathematics., 113(1) (2017), 167-179, doi: 10.12732/ijpam.v113i1.15. 